This is the author version of the following article: Baltimore, Joseph A. (2014). "Modal Realism,

Counterpart Theory, and Unactualized Possibilities." Metaphysica, 15 (1), 209-217. The final publication is available at www.degruyter.com: http://dx.doi.org/10.1515/mp-2014-0013

\title{
Modal Realism, Counterpart Theory, and Unactualized Possibilities
}

\section{Lewis's Modal Realism and Unactualized Possibilities}

According to David Lewis's (1986) modal realism, possible worlds are spatiotemporally and causally isolated entities that are no less real and concrete than ourselves and the world we inhabit. One concern with this view is that it seems unable to capture genuine alternatives to actuality, i.e. unactualized possibilities. The objection, as Lewis (1986, pp. 97-101) considers it, has two main parts. According to the first part, the claim "everything is actual" is analytic:

[I]t is a trivial matter of meaning that whatever there is, is actual. The word 'actual' is a blanket term, like 'entity' or 'exists': it applies to everything...everything without restriction (1986, p. 97).

According to the second part of the objection, this analytic point causes problems for Lewis's modal realism:

Since everything is actual, the other worlds, if such there be, actually exist.

Then it is not merely possible that they exist. They are not unactualised possibilities. In fact they have nothing to do with possibility. For possibility concerns not the far reaches of actuality - not even the reaches of actuality that are spatiotemporally isolated from us, if such there be - but rather it concerns alternatives to actuality. Actuality - all of it, no matter how much of it there is - might have been different, and that is what modality is all about. More of actuality is no substitute for unactualised possibility. (1986, pp. 97-98) 
So, the thrust of the objection is that Lewis's modal realism, which (according to the first part of the objection) simply posits a bunch of actualities, cannot provide any good account of unactualized possibilities or the merely possible, for (according to the second part of the objection) such possibilities are not found in actuality. ${ }^{1}$

In response, Lewis acknowledges the importance of respecting the following bit of common sense: unactualized possibilities are not parts of actuality, they are alternatives to it. ${ }^{2}$ Concerning this thesis, Lewis writes:

Analytic or not, it seems compelling. I too would find it very peculiar to say that modality, as ordinarily understood, is quantification over parts of actuality.

If I were convinced that I ought to call all the worlds actual - in which case also I might be reluctant to call them worlds - then it would become very implausible to say that what might happen is what does happen at some or another world. (1986, p. 100)

And similarly:

...that the modal operators, as ordinarily understood, cannot be quantifiers over subdivisions of actuality...I agree (1986, p. 98).

Thus, there is a motivation underlying the second part of the objection that Lewis agrees with, namely, that unactualized possibilities are not parts of actuality. That is, Lewis concedes that more of actuality is no substitute for unactualized possibility, in the sense that capturing such possibilities requires quantifying over something other than actual entities.

\footnotetext{
${ }^{1}$ For advocates of this objection (in part or whole), see Lewis's references (1986, pp. 88-89). For some more recent supporters of the concern that Lewisian worlds fail to yield unactualized possibilities, see Nathan Salmon (1988) and Michael Jubien (2009, ch. 3).

${ }^{2}$ Sometimes (e.g. 1986, p. 99) Lewis does not explicitly include the qualification "unactualized." It is evident from Lewis's discussion of the thesis, however, that he is concerned with unactualized possibilities. Indeed, otherwise the thesis is plainly false. Clearly some possibilities can be parts of actuality, as is the case with respect to the possibility of you reading this paper. It is unactualized possibilities that are taken to be separate from actuality.
} 
Still, Lewis thinks that he can avoid the thrust of the objection by targeting its first part. Lewis makes clear that he does not take "actual" to be a blanket term applying to everything:

I have already explained that I use it to mean 'this-worldly': it is an indexical, relative term, and as used by us it distinguishes our world and our worldmates from all the other worlds and their inhabitants. (1986, p. 99)

With this understanding of the term "actual," Lewis can apparently keep unactualized possibilities separate from actuality. Relative to our world, all other worlds and their parts are separate from actuality. Therefore, by keeping unactualized possibilities at those other worlds, Lewis's modal realism can respect the claim that unactualized possibilities are not parts of actuality. My primary goal in this paper is to show that, even with Lewis's indexical account of "actual," he cannot so easily keep unactualized possibilities separate from actuality. In the next section, this problem is teased out by considering Lewis's counterpart theory.

\section{Counterpart Theory and the Loss of Postulate 5}

Lewis (1986, pp. 198-220) argues that, on his modal realism, an individual cannot exist in more than one possible world. Having all individuals world-bound, though, raises the concern that, on Lewis's modal realism, no individual has any contingent properties. It seems that an individual, $x$, has a property, $F$, contingently if and only if $x$ is $F$ in the actual world and there is at least one possible world in which $x$ is not $F$. In order to accommodate de re contingency, Lewis (1986, pp. 194-197) appeals to his counterpart theory. An individual, $y$, is a counterpart of $x$ if and only if $y$ sufficiently resembles $x$. Thus, the counterpart relation is not a relation of identity but, rather, a relation of resemblance. According to Lewis, such resemblance enables $y$ to represent $x$ and thereby ground de re modal truths about $x$. So, on Lewis's view, $x$ is contingently 
$F$ if and only if $x$ is $F$ in the actual world and $x$ has a counterpart that is not $F$. I shall refer to the conjunction of Lewis's modal realism and his counterpart theory as "Lewis's modal system."

Among his original eight postulates for counterpart theory, Lewis includes the following, Postulate 5 (P5): nothing is a counterpart of anything else in its world; you have no worldmate counterpart other than yourself. ${ }^{3}$ However, due to the different ways in which one might resolve the vagueness of the counterpart relation, Lewis ultimately abandoned P5. A suitable context might, for example, deliver a counterpart relation on which anything is a counterpart of anything of its kind. Assuming that you belong to the kind human, then not only will you be a counterpart of yourself but so too will every other human in your world. Therefore, the vagueness of the counterpart relation (which allows for rather unselective counterpart relations in certain contexts) makes it implausible to hold P5 as a general postulate after all. ${ }^{4}$

Lewis seems unaware, however, that the loss of P5 undermines the ability of his modal system to keep unactualized possibilities separate from actuality. To see this, suppose that you have a worldmate counterpart distinct from yourself - an option afforded by the rejection of P5-who is not a philosopher. That worldmate counterpart represents you de re as not being a philosopher. But since that worldmate counterpart is part of your world, the corresponding de re possibility of you not being a philosopher is part of your world and, so, part of actuality (relative to your world). Yet you are a philosopher (or at least I will assume so) and, therefore, an alternative way you might have been, an unactualized possibility, is part of actuality (relative to your world). By allowing a worldmate counterpart distinct from yourself, an unactualized de re possibility can hold for you by quantifying solely over actual objects (i.e. you, your world, and

\footnotetext{
${ }^{3}$ Lewis formally presents the postulate in the following way:

P5: $\forall x \forall y \forall z(I x y$ \& Izy \& $C x z$. $\supset x=z)$.

The English readings of the predicates $I x y$ and $C x y$ are " $x$ is in possible world $y$ " and " $x$ is a counterpart of $y$," respectively (Lewis 1968, p.114).

${ }^{4}$ See Lewis (1983, pp. 42-43) and Lewis (1986, p. 232, footnote 22).
} 
your worldmate counterpart, which are all actual objects relative to your world). But, as Lewis admits, it would be implausible to take more of actuality as a substitute for unactualized possibility—one must quantify over something other than actual objects in order to capture such possibilities. Thus, without P5, Lewis's modal system cannot respect the view that unactualized possibilities are not parts of actuality.

It does not help matters to appeal to what Lewis calls "joint possibilities," which are possibilities for ordered sequences of multiple individuals (1986, pp. 232-233). Lewis captures such possibilities by quantifying over sets of individuals, and Lewis (1983, pp. 39-40) makes clear that on his view sets are not, strictly speaking, part of, or in, any possible world. Thus, one might contend, Lewis's account of joint possibilities is not vulnerable to the charge of quantifying only over more of actuality, for sets are not part of any possible world and, therefore, not part of actuality. Notice, though, that sets can still exist from the standpoint of a world, such that "the unit set of a possible individual might only exist from the standpoint of the world that the individual is in" (Lewis, 1983, pp. 39-40). Perhaps, then, one might seek to expand the commonsense thesis in the following way: unactualized possibilities are not parts of actuality, nor do they exist only from the standpoint of actuality. In any event, the appeal to joint possibilities is clearly limited: it does not help with the problematic case described above, in which the relevant de re possibility is for a single individual who exists wholly in actuality. ${ }^{5}$

\footnotetext{
${ }^{5}$ Moreover, note that some possibilities might initially seem to require quantification over sets, when in fact they do not. For example, Lewis (1983, p. 44) applies counterpart theory to the issue of whether a pair of twin brothers might have been born not as twins but, rather, as unrelated inhabitants of separate planets. Instead of taking a set of which the brothers are members, Lewis takes the mereological sum of the pair of brothers (which is wholly in a possible world). Indeed, it would seem that Lewis's general preference for counterpart theory is to quantify only over individuals that are wholly in a possible world: "The language of counterpart theory, and the modal language it replaces, had best be understood as quantifying only over possible individuals [i.e. individuals that are wholly in a possible world]. Modifications are called for if we wish to quantify over more of what there is." (1983, p. 40).
} 
A more far-reaching response is to seek the reinstatement of P5 into Lewis's modal system. Such a move seems rather ad hoc, however, since it follows not from considerations of the nature of the counterpart relation but, rather, a desire to save Lewis's modal system from the problematic consequence of making unactualized possibilities parts of actuality. Moreover, as previously observed, the nature of the counterpart relation-specifically, its vagueness, which allows for rather unselective counterpart relations - suggests that P5 is false. In addition to these concerns, there is a further one, which is the topic of the next section: as we will see, maintaining P5 renders Lewis's modal system vulnerable to difficulties tied to haecceitism.

\section{The Threat of Haecceitism}

Since there are a variety of views associated with the term "haecceitism," Lewis is careful to specify his understanding of the term in the following way:

If two worlds differ in what they represent de re concerning some individual, but do not differ qualitatively in any way, I shall call that a haecceitistic

difference. Haecceitism, as I propose to use the word, is the doctrine that there are at least some cases of haecceitistic difference between worlds. (1986, p. $221)^{6}$

Though Lewis ultimately denies that there are any cases of haecceitistic difference- the reasons for which will be reviewed shortly — he does acknowledge that there seem to be intuitively compelling cases of such differences:

Suppose, for instance, that ours is a world of one-way eternal recurrence with a first epoch but no last. One of the epochs is ours. Which epoch? - there seem to be many possibilities, one of which is the actual one. Perhaps our epoch is in

\footnotetext{
${ }^{6}$ For a helpful discussion of various formulations of haecceitism, including Lewis's, see Bradford Skow (2008).
} 
fact the seventeenth; but we might instead have lived in the 137th epoch. So it seems that there is a possible world that is qualitatively just like ours - the same infinite sequence of epochs, all exactly alike, and exactly like the epochs of our world - but that represents de re, concerning us, that we live in the 137th epoch rather than the seventeenth. Then the difference between that world and ours is a haecceitistic difference. (1986, p. 227)

In this example, we have two worlds that are qualitatively identical. One world, $w$, represents us de re as living in the 17 th epoch, while the other world, $w^{*}$, represents us de re as living in the 137 th epoch. Thus, $w$ and $w^{*}$ differ in what they represent de re without differing qualitatively, which is a haecceitistic difference. And for such intuitive cases of haecceitistic difference, Lewis admits, "It would be very implausible and damaging, I think, just to defy the intuitions" (1986, p. 230). Lewis tries, then, to accommodate the haecceitistic intuition that he might have lived in the 137 th epoch, but without taking on any haecceitistic differences between worlds.

He begins by rejecting the assumption that differences between possibilities are differences between possible worlds. He admits that it is common to take the unit of possibility to be the possible world. However, according to Lewis, while possible worlds are indeed possibilities (the largest or most complete ones), they contain smaller, distinct units of possibility as proper parts. Hence, according to Lewis, "What is not true is that we should count distinct possibilities by counting the worlds that provide them. A single world may provide many possibilities, since many possible individuals inhabit it" (1986, p. 230). ${ }^{7}$

With these smaller units of possibility, Lewis returns to the epoch-world case. Again, take $w$ to be our world in which, it is assumed, we live in the 17th epoch. Since a possibility need

\footnotetext{
${ }^{7}$ For challenges to Lewis's rejection of the thesis that differences between possibilities are always differences between possible worlds, see Sam Cowling (2012) and Jeffrey Russell (2013).
} 
not always be a possible world, $w$ can contain many distinct possibilities. Furthermore, allowing Lewis's worldmate doppelganger in the 137th epoch to be one of Lewis's counterparts, $w$ can include the possibility which represents Lewis de re as living in the 137 th epoch-we need not resort to $w^{*}$. In this way, Lewis seeks to accommodate such de re possibilities "on the cheap," i.e. without taking on any haecceitistic differences between worlds (1986, p. 232).

Clearly, maintaining P5 would put this line of response out of reach. Thus, reinstating P5 in order to avoid making unactualized possibilities parts of actuality only forces Lewis's modal system into another, equally damaging situation: being unable to accommodate, on the cheap, very plausible haecceitistic intuitions. With P5, accommodating those haecceitistic intuitions would require taking on haecceitistic differences between worlds. And as Lewis himself argues, the price of doing so is steep for his modal system: one would need to either accept worldoverlap, which Lewis persuasively argues against, or adopt a non-qualitative counterpart relation, of which Lewis sees no way to make sense (1986, pp. 228-230). ${ }^{8}$

We can also now recognize a serious problem with Lewis's attempt to avoid haecceitism: it fails to respect the claim that unactualized possibilities are not parts of actuality, in the sense that more of actuality is no substitute for unactualized possibility. By seeking a counterpart in the 137th epoch of his own world $w$, Lewis is simply consulting more of actuality, for actuality is "this-worldly" and $w$ is Lewis's world. Therefore, if Lewis is to respect the claim that unactualized possibilities are not parts of actuality, then Lewis cannot account for how he might have been living in the 137 th epoch by looking to a worldmate counterpart in the 137 th epoch. Instead, he would have to consult a world distinct from $w$, such as $w^{*}$, in order to accommodate that de re possibility. But this, of course, is to fall into the clutches of haecceitism.

\footnotetext{
${ }^{8}$ For a recent attempt to make sense of a non-qualitative counterpart relation, see Cowling's (2012) employment of substratum theory.
} 


\section{Fara's Argument}

Before bringing this paper to a close, it is worth considering a related argument made by Delia Graff Fara (2009). Fara also raises concerns about Lewis's strategy for avoiding haecceitism. Fara argues that, when we include an actuality operator $(A C T)$ amongst our expressive resources, Lewis's cheap substitute for haecceitism generates some implausible consequences for counterpart theorists that favor (as Lewis does) a purely qualitative counterpart relation. Returning to the epoch-world case, in which we suppose that our world is a world of one-way eternal recurrence, assume that Fara is in the 17th epoch and (following Lewis's strategy) that she has a worldmate counterpart in the 18th epoch. The following haecceitistic intuition is satisfied:

(1) Fara lives in the 17th epoch $\wedge \diamond$ Fara lives in the 18th epoch.

However, since what goes on in the actual world is actually going on, Fara's worldmate counterpart in the 18th epoch is actually there and, so, represents Fara as actually living in the 18th epoch:

(2) $A C T$ Fara lives in the 17 th epoch $\wedge \diamond A C T$ Fara lives in the 18th epoch.

Then, given that one can infer actually- $P$ from possibly-actually- $P$, we get the following problematic result:

(3) $A C T$ Fara lives in the 17 th epoch $\wedge$ ACT Fara lives in the 18th epoch.

And, to magnify difficulties, Fara takes the actuality operator to commute with conjunction so as to yield the following:

(4) $A C T$ (Fara lives in the 17 th epoch $\wedge$ Fara lives in the 18th epoch).

In what follows, I will contrast Fara's argument with mine. 
According to Fara, the problem with Lewis's strategy for avoiding haecceitism is running into the likes of (3) and (4), while my concern is violating the commonsense thesis that unactualized possibilities are not parts of actuality. Still, one might wonder if these two problems are importantly tied to one another. For example, one might suggest that the reason why unactualized possibilities are not parts of actuality is because allowing them to be so would result in the likes of (3) and (4). There are reasons to reject such a suggestion, however. Though Fara's argument might be used to reinforce the claim that unactualized possibilities are not parts of actuality, it is far from clear that the force of the latter reduces to that of the former. Notice that there is no apparent inconsistency between resisting the inclusion of an actuality operator amongst our expressive resources, and still maintaining that unactualized possibilities are not parts of actuality. Moreover, one might take the plausibility of the claim that unactualized possibilities are not parts of actuality to be grounded in basic intuition. Lewis, after all, seems content with the inherent intuitive appeal of the claim, respecting it as a thesis of common sense.

This brings us to a further difference between my argument and Fara's. Fara's argument relies on introducing an actuality operator, which is foreign to Lewis's modal system and not even addressed in his scheme for translating the language of quantified modal logic into the language of counterpart theory. In contrast, the claim that unactualized possibilities are not parts of actuality is explicitly accepted by Lewis. Thus, my approach tackles Lewis's modal system from within, exploiting intuitions to which Lewis himself conceded authority.

In closing, let us take stock. We have seen how the addition of counterparty theory (without P5) to modal realism undermines its ability to respect the claim that unactualized possibilities are not parts of actuality. And simply reinstating P5 would be a bad move, since doing so would (i) look ad hoc, (ii) be implausible given that there can be rather unselective 
counterpart relations, and (iii) undermine Lewis's ability to accommodate haecceitistic intuitions on the cheap. Furthermore, we have uncovered a problem for Lewis's attempt to avoid haecceitism: it involves quantifying solely over actual objects in order to capture unactualized possibilities, which is the very sort of thing that Lewis himself confessed to be problematic. More of actuality is no substitute for unactualized possibility.

Acknowledgements: Thanks to Sam Cowling and Adam Podlaskowski for helpful feedback on earlier drafts of this paper.

\section{References}

Cowling, S. (2012) Haecceitism for Modal Realists. Erkenntnis, 77: 399-417.

Fara, D. G. (2009) Dear Haecceitism. Erkenntnis, 70: 285-297.

Jubien, M. (2009) Possibility. Oxford: Oxford University Press.

Lewis, D. (1968) Counterpart Theory and Quantified Modal Logic. Journal of Philosophy, 65:

113-126. Reprinted with postscripts in his Philosophical Papers, Volume I (1983) pp. 2646.

Lewis, D. (1983) Philosophical Papers, Volume I. Oxford: Oxford University Press.

Lewis, D. (1986) On the Plurality of Worlds. Oxford: Basil Blackwell.

Russell, J. (2013) Possible Worlds and the Objective World. Philosophy and Phenomenological Research: DOI: 10.1111/phpr.12052.

Salmon, N. (1988) An Empire of Thin Air. The Philosophical Review, XCVII: 237-244. Reprinted in his Metaphysics, Mathematics, and Meaning: Philosophical Papers I (2006) Ch. 6 . 
Salmon, N. (2006) Metaphysics, Mathematics, and Meaning: Philosophical Papers I. Oxford: Oxford University Press.

Skow, B. (2008) Haecceitism, Anti-Haecceitism, and Possible Worlds. The Philosophical Quarterly, 58: 97-107. 